\title{
Active control of friction by transverse oscillations
}

\author{
J. BENAD ${ }^{1}$, K. NAKANO ${ }^{2, *}$, V. L. POPOV ${ }^{1,3, *}$, M. POPOV ${ }^{1,3,4}$ \\ ${ }^{1}$ Technische Universität Berlin, Berlin 10623, Germany \\ ${ }^{2}$ Yokohama National University, Yokohama 240-8501, Japan \\ ${ }^{3}$ National Research Tomsk Polytechnic University, Tomsk 634050, Russia \\ ${ }^{4}$ National Research Tomsk State University, Tomsk 634050, Russia \\ Received: 06 September 2017 / Accepted: 24 November 2017 \\ (C) The author(s) 2018. This article is published with open access at Springerlink.com
}

\begin{abstract}
The present paper is devoted to a theoretical analysis of sliding friction under the influence of in-plane oscillations perpendicular to the sliding direction. Contrary to previous studies of this mode of active control of friction, we consider the influence of the stiffness of the tribological contact in detail and show that the contact stiffness plays a central role for small oscillation amplitudes. In the present paper we consider the case of a displacement-controlled system, where the contact stiffness is small compared to the stiffness of the measuring system. It is shown that in this case the macroscopic coefficient of friction is a function of two dimensionless parameters - a dimensionless sliding velocity and dimensionless oscillation amplitude. In the limit of very large oscillation amplitudes, known solutions previously reported in the literature are reproduced. The region of small amplitudes is described for the first time in this paper.
\end{abstract}

Keywords: sliding friction; in-plane oscillation; contact stiffness; coefficient of friction; active control of friction

\section{Introduction}

The interrelation of oscillations and friction is an old problem with fundamental importance for the understanding of friction and for countless practical applications. From the physical point of view, friction is fundamentally a non-stationary process. Brillouin [1] pointed out as early as 1899 that dry friction can occur at low velocities only due to elastic instabilities on the microscale. Vibrations can strongly influence friction [2] and friction often leads to vibrational instabilities [3]. Thus, friction should always be understood as the interplay of dynamics and friction on different spatial and temporal scales. This interplay has many particular aspects which have been studied intensively in the past decades: (I) Influence of vibrations on friction was studied, e.g., in Refs. [4-6]. (II) Frictionally induced oscillations have been studied, e.g., in Refs. [3, 7-9]. (III) The interaction of self-excited vibrations and friction was subject of studies [10-12]. (IV) The interplay of vibrations and oscillations is a central principle of oscillation-based actuation [13-17]. (V) Finally, oscillations may lead to energy dissipation, which of course is intimately connected with all other above mentioned points in Refs. [18-20].

The present paper is devoted exclusively to the aspect (I) of the above list-the direct influence of oscillations on friction. Studies of this influence started in the late 50s and 60s of the 20th century [21-25]. Most models used for the analysis of the active control of friction were based on the study of dynamics of rigid bodies. Only recently it was recognized that the deformability of the bodies and especially the contact stiffness plays a central role in determining the frictional behavior at small oscillation amplitudes and small sliding velocities [26, 27, 6]. However, of the three possible oscillation directions: (a) out-ofplane, (b) in-plane perpendicular to sliding, (c) in-plane

* Corresponding authors: K. NAKANO, E-mail: nakano@ynu.ac.jp; V. L. POPOV, E-mail: v.popov@tu-berlin.de 
in sliding direction-only the influence of out-of-plane oscillations has been studied in detail so far [28, 29]. Complete studies of friction under in-plane oscillations - both in the sliding direction and perpendicular to the sliding direction-are yet to be performed.

The present paper partially closes this gap and provides an analysis of friction under transverse oscillations-in-plane oscillations perpendicular to the sliding direction. The particular interest in this mode of active control of friction is partly due to the recently demonstrated importance of transverse oscillations both for the stability of macroscopic sliding and the design of robust tribological measurement techniques [30-33].

To achieve qualitative understanding of the corresponding phenomena, we follow the strategy already used in a recent analysis of out-of-plane oscillations $[28,37]$ : we start with a very simple model, where the contact is modeled as a single spring with constant normal and tangential stiffness in a displacementcontrolled setting (i.e., with a very stiff surrounding system). System-dynamical "feedback" from the contact to the surrounding system is thereby neglected.

\section{Simplified one-spring model}

Let us consider an elastic body that is brought into contact with a flat elastic substrate and then subjected to a superposition of horizontal movement with a constant velocity and sideways oscillations. In the contact of elastic bodies, both normal and tangential contact problems can be reduced to a contact of an elastic body and a rigid substrate with renormalized elastic coefficients [34]. In this paper we further reduce the elastic body to a single spring with some normal stiffness (the magnitude of which does not play any role in the present study) and tangential stiffness $k$. We assume that between the spring and the plane, there is a friction force described by the simplest form of Coulomb's law of friction $[35,36]$ with a constant coefficient of friction $\mu_{0}$. A schematic drawing of the model is shown in Fig. 1: the "body" (the upper point $\mathrm{P}$ of the spring) is forced to move with a constant velocity $v_{0}$ in the $x$-direction, and also to perform a harmonic oscillation in the $y$-direction according to $y_{\mathrm{P}}=y_{0} \sin \omega t$.

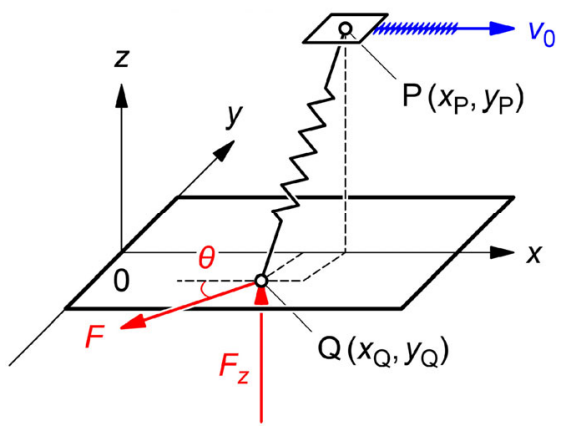

Fig. 1 Schematic representation of the considered system: An elastic body modeled as a spring is forced into a controlled movement at the upper point $\mathrm{P}$, while the immediate contact point Q follows according to the equilibrium conditions. It is assumed that between the contact point and the horizontal plane there is a force of friction described by the classical Coulomb law.

While the time-resolved reaction of the instantaneous friction force on the loading history is also of interest, in this paper we consider exclusively the forces in the steady state, averaged over one period of oscillation. In this connection it is important to lay down the terminology used in the paper: all processes referring to the time scale much smaller than the period of one oscillation are considered here as "microscopic" while the processes and quantities running or defined on the time scale much larger than the period of one oscillation are called "macroscopic". Our goal is to determine the macroscopic values of normal and tangential forces (meaning their average values over one oscillation period) and the corresponding macroscopic coefficient of friction. The above-mentioned timeresolved reaction, on the contrary, refers to the microscopic scale; it will be considered in a separate paper.

Figure 2 shows the system projected onto the contact plane $(x, y)$ for the cases when the immediate contact point sticks (Fig. 2(a)) and for the sliding state (Fig. 2(b)). In the sticking state, the velocity of the "foot point" $Q$ is zero.

As the vector of the spring force is determined uniquely by two quantities: the elongation $l$ and the inclination angle $\theta$ to the direction of the macroscopic movement, it is convenient to write equations in terms of these two quantities.

The coordinates of the upper end of the spring can be written as

$$
\begin{aligned}
& x_{\mathrm{P}}=v_{0} t \\
& y_{\mathrm{P}}=y_{0} \sin \omega t
\end{aligned}
$$




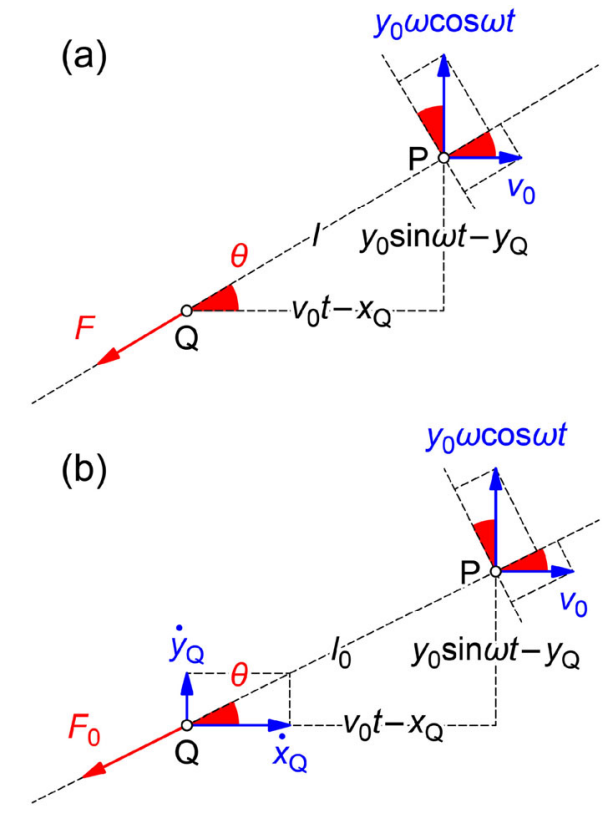

Fig. 2 Projection of the considered system onto the $(x-y)$-plane. (a) stick phase; (b) sliding phase.

and the corresponding velocities

$$
\begin{aligned}
& \dot{x}_{\mathrm{P}}=v_{0}, \\
& \dot{y}_{\mathrm{P}}=y_{0} \omega \cos \omega t
\end{aligned}
$$

Sticking phase: if the immediate contact point is sticking then the equations for the angle $\theta$ and the length $l$ are

$$
\begin{aligned}
& \dot{\theta}=\frac{y_{0} \omega \cos \omega t \cos \theta-v_{0} \sin \theta}{l} \\
& \dot{i}=y_{0} \omega \cos \omega t \sin \theta+v_{0} \cos \theta
\end{aligned}
$$

where $y_{0} \omega \cos \omega t \cos \theta-v_{0} \sin \theta$ is the component of the velocity of the point $\mathrm{P}$ in the direction perpendicular to the elongation $l$ and $y_{0} \omega \cos \omega t \sin \theta+v_{0} \cos \theta$ is the velocity component of the same point in the direction of the elongation (see Fig. 2(a) for illustration).

These equations remain valid as long as the elongation $l$ remains smaller than the critical value

$$
l<l_{0}=\frac{\mu_{0} F_{z}}{k}
$$

Slipping phase: after the elongation $l$ reaches the critical value $l_{0}$, it does not increase further, but remains equal to $l_{0}$. Note that due to the equilibrium conditions in the immediate contact point $Q$, its movement occurs always in the direction of the elongation. Thus it has no velocity component perpendicular to the direction of $l$. The angular velocity of the direction of the elongation $l$ is given by the ratio of the difference of the transversal velocity components of points $\mathrm{P}$ and $\mathrm{Q}$ to the (constant) length $l_{0}$. However, as the transversal component of velocity of point $Q$ is zero, Eq. (3) remains valid, except that $l$ has to be replaced by $l_{0}$ :

$$
\dot{\theta}=\frac{y_{0} \omega \cos \omega t \cos \theta-v_{0} \sin \theta}{l_{0}}
$$

This equation remains valid as long as the projection of the velocity of point $\mathrm{P}$ on the direction of $l$ remains positive:

$$
y_{0} \omega \cos \omega t \sin \theta+v_{0} \cos \theta>0
$$

This condition guarantees that the point $Q$ is following $\mathrm{P}$ in the direction of the elongation. Otherwise it stops until the condition Eq. (5) is fulfilled again.

Introducing dimensionless variables and operators

$$
\tau=\omega t, \quad()^{\prime}=\frac{\mathrm{d}}{\mathrm{d} \tau}, \quad \tilde{y}_{0}=\frac{y_{0}}{l_{0}}, \tilde{l}=\frac{l}{l_{0}}, \tilde{v}_{0}=\frac{v_{0}}{l_{0} \omega}
$$

one can rewrite Eqs. (3) and (4) as

$$
\begin{gathered}
\theta^{\prime}=\frac{1}{\tilde{l}}\left(-\tilde{v}_{0} \sin \theta+\tilde{y}_{0} \cos \theta \cos \tau\right), \text { for stick phase } \\
\tilde{l}^{\prime}=\tilde{y}_{0} \sin \theta \cos \tau+\tilde{v}_{0} \cos \theta, \text { for stick phase }
\end{gathered}
$$

and Eqs. (6) and (7) as

$$
\begin{gathered}
\theta^{\prime}=-\tilde{v}_{0} \sin \theta+\tilde{y}_{0} \cos \theta \cos \tau, \quad \text { for slip phase } \\
\tilde{y}_{0} \sin \theta \cos \tau+\tilde{v}_{0} \cos \theta>0 . \quad \text { for slip phase }
\end{gathered}
$$

The goal of our study is to determine the average force component in the sliding direction

$$
\left\langle F_{x}\right\rangle=\langle k \cdot l \cdot \cos \theta\rangle=\mu_{0} F_{z}\langle\tilde{l} \cdot \cos \theta\rangle
$$

(where $\langle\ldots\rangle$ denotes averaging over one period of oscillation in the steady state) and the corresponding macroscopic coefficient of friction defined as

$$
\mu_{\text {macro }}=\frac{\left\langle F_{x}\right\rangle}{F_{z}}=\mu_{0}\langle\tilde{l} \cdot \cos \theta\rangle
$$


or the normalized coefficient of friction

$$
\tilde{\mu}_{\text {macro }}=\frac{\mu_{\text {macro }}}{\mu_{0}}=\langle\tilde{l} \cdot \cos \theta\rangle
$$

Note that all the Eqs. (9)-(12) as well as definition, Eq. (15), depend only on two dimensionless parameters $\tilde{y}_{0}$ and $\tilde{v}_{0}$. We therefore present all the results of this paper as function of these parameters.

\section{Static coefficient of friction}

If the system starts from the neutral state with zero tangential force and moves slowly in the $x$-direction, while at the same time oscillating in the $y$-direction with amplitude $y_{0}$ (see Fig. 3 ), then the macroscopically seen (average) spring force will be increasing until the critical state shown in Fig. 3 is reached. In this state, the component of the spring force in the $x$-direction is equal to $F_{x}=\mu_{0} F_{z} \sqrt{1-y_{0}^{2} / l_{0}^{2}}$ and remains unchanged during the entire oscillation cycle.

Thus, the average coefficient of friction in this state, which we can interpret as the static coefficient of friction is given by

$$
\tilde{\mu}_{\text {macrostatic }}=\sqrt{1-y_{0}^{2} / l_{0}^{2}}=\sqrt{1-\tilde{y}_{0}^{2}}
$$

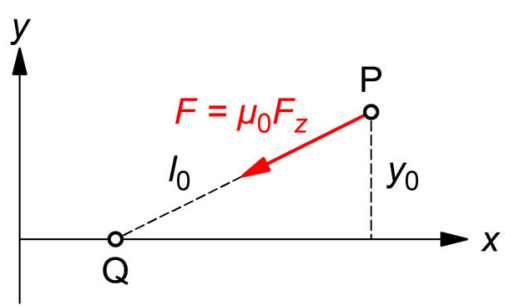

Fig. 3 Critical state of a system with oscillation amplitude $y_{0}$ and very slow motion in the $x$-direction.

\section{Continuous sliding and stick-slip motion}

It is intuitively clear that at sufficiently high sliding velocities $v_{0}$, the contact point will be in the sliding state all the time, while at smaller sliding velocities the motion will consist of a sequence of stick and slip phases. On the parameter plane $\left(\tilde{v}_{0}, \tilde{y}_{0}\right)$, the region of continuous sliding is separated from the stick-slip region by a boundary that can be found numerically by solving Eq. (11), which is valid in the region of continuous sliding, and checking the fulfillment of the condition (12). Figure 4 shows the areas of the continuous and intermittent sliding and the boundary line between them.

\subsection{Small oscillation amplitudes $\tilde{y}_{0} \ll<1$}

As can be seen from Eq. (11), in this case the angle also remains small, so that we can set in Eq. (11) $\sin \theta \approx \theta$ and $\cos \theta \approx 1$ :

$$
\theta^{\prime}+\tilde{v}_{0} \theta=\tilde{y}_{0} \cos \tau
$$

The steady-state solution of this equation is given by

$$
\theta=\frac{\tilde{y}_{0}}{\sqrt{1+\tilde{v}_{0}^{2}}} \cos (\tau+\varphi)
$$

with

$$
\tan \varphi=-1 / \tilde{v}_{0}
$$

This equation is valid for small angles, i.e.

$$
\frac{\tilde{y}_{0}}{\sqrt{1+\tilde{v}_{0}^{2}}}<<1
$$

The borderline between small and large angles is shown in Fig. 4 by a dashed line. Substituting Eq. (18) into the condition for continuous sliding, Eq. (12), we can rewrite this condition as

$$
\frac{\tilde{y}_{0}^{2}}{\sqrt{1+\tilde{v}_{0}^{2}}} \cos (\tau+\varphi) \cos \tau+\tilde{v}_{0}>0
$$

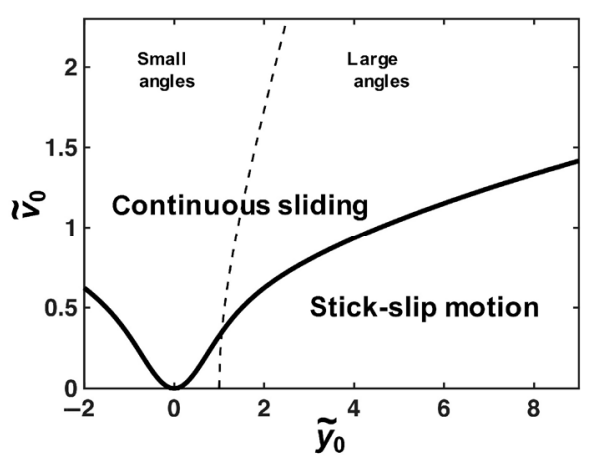

Fig. 4 Area of continuous sliding and area of stick-slip motion (separated by the bold line) over the two system parameters. Also shown are the regions of small and large angles according to Eq. (20) (dashed line). 
or

$$
\frac{\tilde{y}_{0}^{2}}{2 \sqrt{1+\tilde{v}_{0}^{2}}} \cos (2 \tau+\varphi)+\tilde{v}_{0}+\frac{\tilde{y}_{0}^{2} \tilde{v}_{0}}{2\left(1+\tilde{v}_{0}^{2}\right)}>0
$$

It is fulfilled for any $\tau$ if

$$
\tilde{y}_{0}^{2}<2 \tilde{v}_{0}\left(1+\tilde{v}_{0}^{2}\right)\left[\sqrt{1+\tilde{v}_{0}^{2}}+\tilde{v}_{0}\right]
$$

Expanding the right-hand-side of Eq. (23) up to the terms of second order in $\tilde{v}_{0}$, we obtain the condition $\tilde{y}_{0}^{2}=2 \tilde{v}_{0}\left(1+\tilde{v}_{0}\right)$ for the border line. Solving it with respect to $\tilde{v}_{0}$ gives

$$
\tilde{v}_{0, \text { crit }}=\sqrt{\frac{1}{4}+\frac{1}{2} \tilde{y}_{0}^{2}}-\frac{1}{2}
$$

This limiting case is displayed in Fig. 5.

\subsection{Large oscillation amplitudes $\tilde{y}_{0}>1$}

In this case the motion occurs almost perpendicular to the direction of the average velocity and during most of the oscillation period the contact is sliding. Only in the vicinity of the "turning points" there arises the possibility of stick, because both components of the driving velocity become small. The first turning point corresponds to $\tau=\pi / 2$. Introducing a new variable $\xi=\tilde{v}_{0}(\tau-\pi / 2)$, we can rewrite Eq. (11) as

$$
\frac{\mathrm{d} \theta}{\mathrm{d} \xi}=-\sin \theta-\frac{\tilde{y}_{0}}{\tilde{v}_{0}{ }^{2}} \xi \cos \theta
$$

The behavior described by this equation depends on the single parameter $\tilde{y}_{0} / \tilde{v}_{0}^{2}$. Its numerically

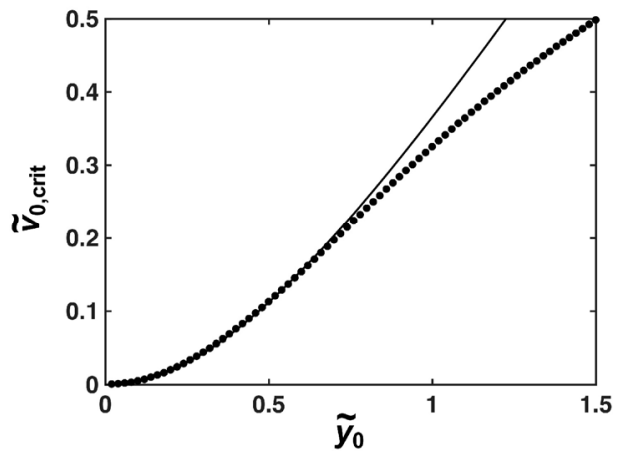

Fig. 5 Approximation of the numerical results for the border line (dots) with Eq. (24) (solid line) for small oscillation amplitudes. determined critical value is $\tilde{y}_{0} / \tilde{v}_{0}^{2} \approx 4.5$. Thus for the critical velocity we get

$$
\tilde{v}_{0, \text { crit }} \approx \frac{1}{\sqrt{4.5}} \tilde{y}_{0}^{1 / 2} \approx 0.47 \cdot \tilde{y}_{0}^{1 / 2}
$$

While this equation describes the asymptotic behavior very well, a slightly more complex equation can be constructed to approximate the complete dependence, both for small and large oscillation amplitudes:

$$
\tilde{v}_{0, \text { crit }}=\frac{0.47 \tilde{y}_{0}^{5 / 2}}{\tilde{y}_{0}{ }^{2}+\frac{0.94 \tilde{y}_{0}^{1 / 2}}{1+\tilde{y}_{0}{ }^{2}}}
$$

This approximation is shown in Fig. 6 together with Eq. (26) and the numerical results.

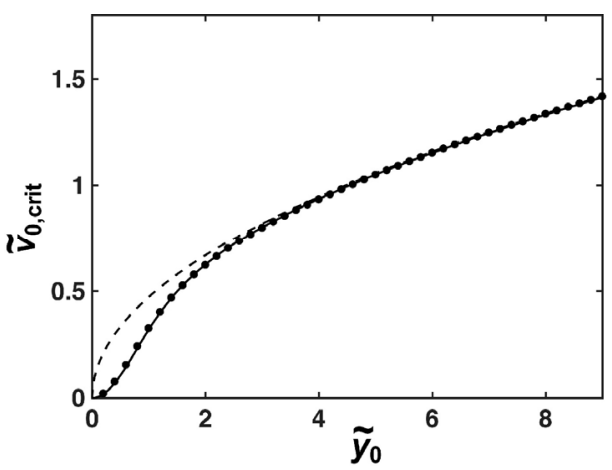

Fig. 6 Approximation of the numerical results for the border line (dots) with relation Eq. (26) (dashed line) and relation Eq. (27) (solid line).

\section{The macroscopic coefficient of friction over the entire parameter space}

For each point $\left(\tilde{v}_{0}, \tilde{y}_{0}\right)$ in the parameter plane, there is a macroscopic coefficient of friction $\tilde{\mu}_{\text {macro }}$. It is displayed over the entire parameter space in Fig. 7.

In the Fig. 8 the same dependence is shown by cuts of the plot in Fig. 7 along the $\tilde{v}_{0}$-axis.

While the exact quantitative description of the coefficient of friction on the entire parameter plane is complicated, the general structure of the dependence is relatively simple and is determined by a small number of "cornerstone" features. The general classification of various behaviors is very similar to that 


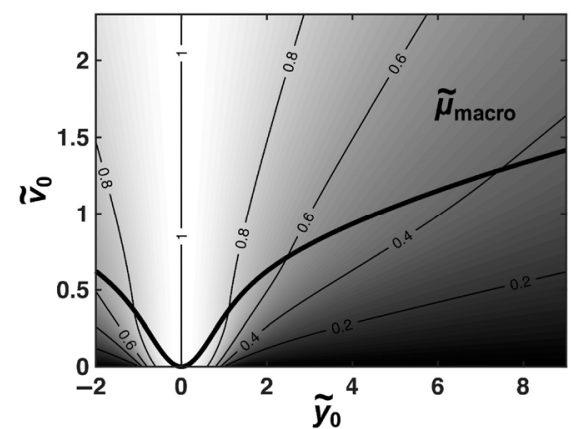

Fig. 7 The macroscopic coefficient of friction displayed over the two system parameters. The region of continuous sliding is above the bold line.

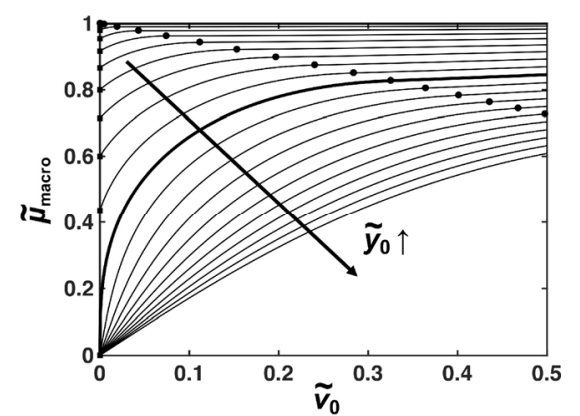

Fig. 8 The lines show the macroscopic coefficient of friction over the dimensionless velocity. They represent vertical cuts through the surface shown in Fig. 7. The graphs are shown for $\tilde{y}_{0}=0,0.1,0.2,0.3,0.4, \ldots, 2$. The amplitude $\tilde{y}_{0}=1$ is highlighted with the bold solid line. The round dots represent the border line, here indicating the critical macroscopic coefficient of friction over the critical velocity. The squares mark the static coefficient of friction as given by Eq. (16).

given in Ref. [37] for the case of normal oscillations:

- Without oscillations, the macroscopic coefficient of friction is constant and equal to its microscopic value $\mu_{0}$, thus $\tilde{\mu}_{\text {macro }}=1$.

- With increasing oscillation amplitude, the static coefficient of friction decreases according to Eq. (16) and vanishes at $\tilde{y}_{0}=1$ (bold line in Fig. 8).

- Further increase of the oscillation amplitude leads to further decrease of the macroscopic coefficient of friction at finite sliding velocities while the static coefficient of friction remains zero.

- The qualitative behavior of the coefficient of friction as a function of velocity is different for the cases of small $\left(\tilde{y}_{0}<<1\right)$ and large $\left(\tilde{y}_{0}>>1\right)$ oscillation amplitudes:

(1) In the region of small oscillation amplitudes, the coefficient of friction is roughly speaking increasing monotonically from its static value to the value corresponding to the point of continuous sliding. After this point, the coefficient of friction increases very slowly and can be approximately assumed to be constant. The critical velocity of continuous sliding thus retains at least approximately the meaning of the "critical velocity of controllability" of friction introduced in Ref. [37]. A more detailed analysis of this range of oscillation amplitudes, which is of most interest to applications, is provided in the next section.

(2) In the region of large oscillation amplitudes, the differentiation between the cases of continuous sliding and intermittent sliding loses its importance, so that one can define a law of friction that is valid with good accuracy in the whole range of sliding velocities. A detailed analysis is given in the next Section. Note that this case was the only one considered in the earlier studies of the influence of sideways oscillations on friction Refs. [4, 5, 2].

\section{Coefficient of friction at low and high oscillation amplitudes}

\subsection{Coefficient of friction at low oscillation amplitudes}

The simple structure of the frictional law in the region of small oscillation amplitudes, $\tilde{y}_{0}<<1$, is illustrated in Fig. 9, where the dependencies of the coefficient of friction on the velocity are shown in normalized variables: the deviation of the macroscopic coefficient

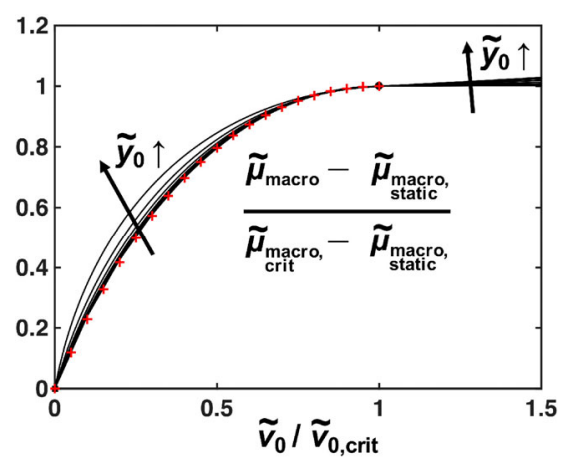

Fig. 9 Displayed are transformed lines from Fig. 8 so that the square markers of the static friction coefficient and the round markers of the critical friction coefficient lie on top of each other. Displayed in this figure are only lines that lie above the bold line in Fig. 8: $\tilde{y}_{0}=0.1,0.2,0.3, \ldots, 0.9$, the bold line itself is not displayed here. The approximation Eq. (28) of this "master curve" is shown with red crosses. 
of friction from its static value normalized by the difference between the value of the border line between stick-slip and continuous sliding vs. velocity normalized by the critical velocity of continuous sliding.

One can see that at small oscillation amplitudes these dependences collapse with acceptable accuracy to a single "master curve", which can be approximated with

$\frac{\tilde{\mu}_{\text {macro }}-\tilde{\mu}_{\text {macro,static }}}{\tilde{\mu}_{\text {macro,crit }}-\tilde{\mu}_{\text {macro,static }}}=1-\left[\frac{3}{4}\left(\frac{\tilde{v}_{0}}{\tilde{v}_{0, \text { crit }}}-1\right)^{2}+\frac{1}{4}\left(\frac{\tilde{v}_{0}}{\tilde{v}_{0, \text { crit }}}-1\right)^{4}\right]$

which coincides with the velocity dependence in the case of out-of-plane oscillations considered in Ref. [28] (shown with red crosses in Fig. 9). After passing the critical value of velocity the coefficient of friction changes only very slowly.

Thus, for small oscillation amplitudes the "law of friction" is completely determined by the value of the static coefficient of friction, Eq. (16), the value of the coefficient of friction in the critical state and the more or less universal transition between both points, Eq. (28).

From the above-mentioned three determining parameters of the law of friction at low oscillation amplitudes, static coefficient of friction, critical velocity of continuous sliding and coefficient of friction at the critical velocity, two are already known and given by Eq. (16) and Eq. (27), respectively. We now consider the macroscopic coefficient of friction $\tilde{\mu}_{\text {macro,crit }}$ directly on the border line. For very low values of $\tilde{y}_{0}$, the coefficient of friction in the area of continuous sliding (including the border line) can be calculated by substituting Eq. (18) into Eq. (15):

$$
\tilde{\mu}_{\text {macro }}=\langle\cos (\kappa \cos (\tau+\varphi))\rangle
$$

with

$$
\kappa=\frac{\tilde{y}_{0}}{\sqrt{1+\tilde{v}_{0}^{2}}}
$$

Expanding Eq. (29) up to the second power of $\kappa$ gives

$$
\tilde{\mu}_{\text {macro }}=1-\frac{\kappa^{2}}{2}\left\langle\cos ^{2}(\tau+\varphi)\right\rangle=1-\frac{1}{4} \frac{\tilde{y}_{0}^{2}}{\left(1+\tilde{v}_{0}^{2}\right)}
$$

For large velocities, the coefficient of friction tends to the limiting value $\tilde{\mu}_{\text {macro }}=1$, as it should. At the borderline defined by $\tilde{v}_{0} \approx \tilde{y}_{0}^{2} / 2$ in the first approximation we get

$$
\tilde{\mu}_{\text {macro,crit }}=1-\frac{\tilde{y}_{0}^{2}}{4}
$$

\subsection{Coefficient of friction at high oscillation amplitudes}

In the case $\tilde{y}_{0}>1$, for most of the oscillation cycle the contact point $Q$ is in the sliding state with the possible exception of "turning points" which, however, do not substantially influence the average coefficient of friction. In this case, in Eq. (11), the derivative on the left-hand-side can be neglected compared with the terms on the right-hand side and this equation can be written as

$$
0=-\tilde{v}_{0} \sin \theta+\tilde{y}_{0} \cos \theta \cos \tau
$$

Hence,

$$
\tan \theta=\frac{\tilde{y}_{0}}{\tilde{v}_{0}} \cos \tau
$$

For the macroscopic coefficient of friction, we obtain, using Eq. (15):

$$
\begin{aligned}
& \tilde{\mu}_{\text {macro }}=\langle\cos \theta\rangle=\left\langle\frac{1}{\sqrt{1+\tan ^{2} \theta}}\right\rangle \\
& =\frac{1}{2 \pi} \int_{0}^{2 \pi} \frac{\mathrm{d} \tau}{\sqrt{1+\left(\frac{\tilde{y}_{0}}{\tilde{v}_{0}}\right)^{2} \cos ^{2} \tau}}=\frac{2}{\pi} K\left(i \frac{\tilde{y}_{0}}{\tilde{v}_{0}}\right)
\end{aligned}
$$

where

$$
K(\xi)=\int_{0}^{\pi / 2} \frac{\mathrm{d} \tau}{\sqrt{1-\xi^{2} \sin ^{2} \tau}}
$$

is the complete elliptic integral of the first kind. The dependence Eq. (35) is shown in Fig. 10. This dependence reproduces the results of earlier studies of this mode of active control of friction in Refs. [4, 5, 2]. Equation (35) shows that the coefficient of friction at large oscillation amplitudes is a function of a single 


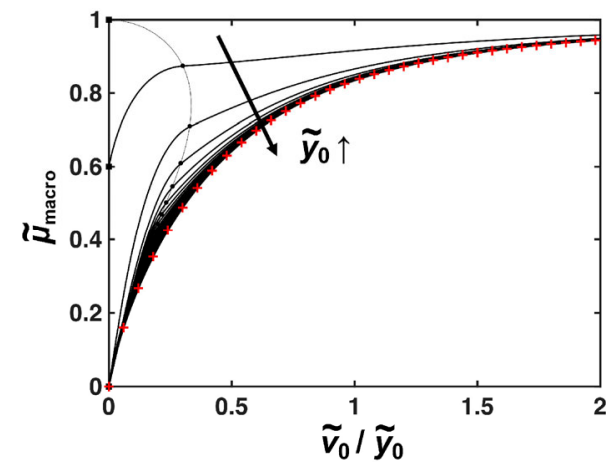

Fig. 10 Dependence of the coefficient of friction on the $\tilde{v}_{0} / \tilde{y}_{0}$ -ratio shown for oscillation amplitudes $\tilde{y}_{0}=0,0.8,1.6,2.4, \ldots$ all the way up to very high values of $\tilde{y}_{0}=80$. The results converge to relation Eq. (35), which is shown with red crosses. Also displayed is the border line (thin solid line with black dots).

parameter combination $\frac{\omega y_{0}}{v_{0}} \frac{\tilde{y}_{0}}{\tilde{v}_{0}}=\frac{\omega y_{0}}{v_{0}}$. Figure 10 shows that the dependences of the normalized coefficient of friction on the parameter $\tilde{y}_{0} / \tilde{v}_{0}$ really do tend towards the "master curve" given by Eq. (35) (red crosses in Fig. 10).

\section{Summary}

We presented a general theoretical analysis of the influence of transverse oscillations on the macroscopically observed coefficient of friction. Unlike previous works, we explicitly took into account the contact stiffness. The natural length scale of the system is the elongation $l_{0}$ at which sliding starts, which depends on the normal force, the contact stiffness and the coefficient of friction according to Eq. (5). The natural scale of velocity is given by the sliding velocity $v_{0}$. Oscillation introduces an additional variable having the dimension of length - the oscillation amplitude, $y_{0}$, and an additional quantity having the dimension of velocity, $l_{0} \omega$. We have found that the dependence of the coefficient of friction on velocity is completely determined by two dimensionless parameters: the dimensionless amplitude of oscillation $\tilde{y}_{0}=y_{0} / l_{0}$ given by the ratio of the above two characteristic lengths; and dimensionless velocity, $\tilde{v}_{0}=v_{0} / l_{0} \omega$.

Figure 11 summarizes schematically the main findings of the present paper. Contrary to the previous figures, we use the non-normalized coefficient of

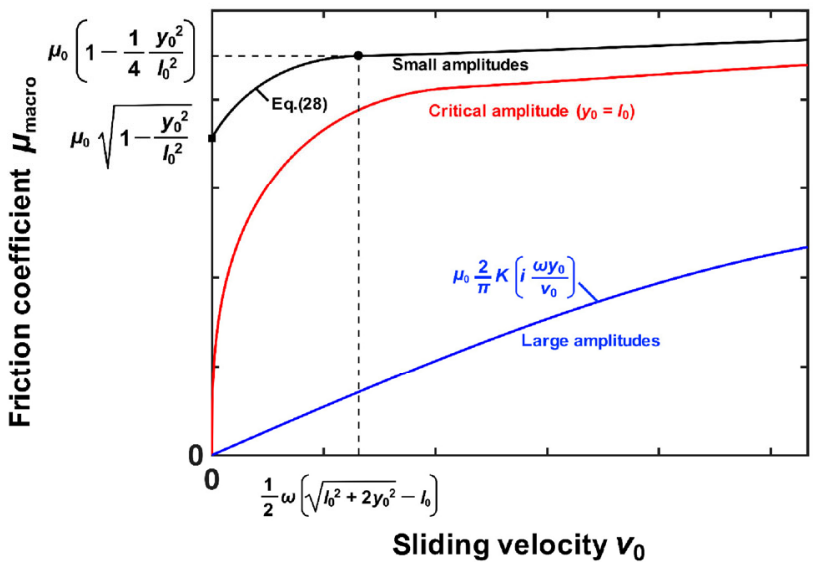

Fig. 11 Schematic representation of the law of friction (dependence of the friction coefficient on the macroscopic sliding velocity).

friction and the non-normalized sliding velocity $v_{0}$, as this better highlights the main tendencies and is easier to compare with experiment.

As in the case of out-of-plane oscillations discussed in Ref. [33], there is qualitatively different behavior in the case of oscillation amplitudes smaller than some critical value (which in the present case is given by $y_{0}=l_{0}$ ) and in the case of large oscillation amplitudes.

In the case of large amplitudes, the behavior is relatively simple and coincides with the well-known solution obtained in Ref. [4] and later in Ref. [5] which, however, never could be fitted to experimental results Ref. [15]. In this case the static friction force is identically zero and the coefficient of friction is increasing monotonically according to the more or less universal law given by Eq. (35) tending to the microscopic value in the limit of very high velocities. In dimensional variables it reads:

$$
\mu_{\text {macro }}=\mu_{0} \frac{2}{\pi} K\left(i \frac{\omega y_{0}}{v_{0}}\right)
$$

In the case of small oscillation amplitudes, there is a final static friction coefficient. In this region, the law of friction is roughly determined by three parameters: the static coefficient of friction, Eq. (16) or in non-normalized form:

$$
\mu_{\text {macro,static }}=\mu_{0} \sqrt{1-y_{0}^{2} / l_{0}^{2}}
$$

the critical velocity of continuous sliding, Eq. (24) which in dimensional variables reads 


$$
v_{0, \text { crit }}=\frac{1}{2} \omega\left[\sqrt{l_{0}^{2}+2 y_{0}^{2}}-l_{0}\right]
$$

and the coefficient of friction at this velocity, Eq. (32):

$$
\mu_{\text {macro, } \text { rrit }}=\mu_{0}\left(1-\frac{1}{4} \frac{y_{0}^{2}}{l_{0}^{2}}\right)
$$

At larger velocities, the coefficient of friction has a very slowly changing plateau.

Let us briefly discuss the physical mechanism of the reduction of friction by transverse oscillations. In the case of out-of-plane oscillations, this reduction is exclusively due to the stick-slip motion: during the stick-phase the force of friction is smaller than the sliding frictional force; therefore, the average frictional force is smaller than the force at stationary sliding [28]. In the case of transverse oscillations, there are two main causes of friction reduction: (a) the occurrence of phases of stick and (b) the deflection of the local force of friction in the contact point from the direction of the macroscopic sliding. The first of these mechanisms is common for all kinds of active control of friction by oscillations. The second one is characteristic only for the case of transverse oscillations considered in the present paper. While the absolute value of the sliding force remains constant, the macroscopic coefficient of friction is determined by the projection of the force on the direction of the macroscopic sliding which in the case of transverse oscillations does not coincide with the direction of macroscopic sliding. Thus, it is always reduced compared to the absolute value of the sliding friction by the average value of $\cos \theta$, where $\theta$ is the angle between the sliding direction and the direction of the instant force of friction. This mechanism manifests itself in Eq. (15). Due to this second mechanism, the reduction of friction occurs even in the cases of continuous sliding.

\section{Outlook}

In the future, several problems have to be considered that have not been studied yet. From the three basic oscillation directions till now only two have been studied in detail, with account of the contact stiffnessthe out-of-plane oscillations [28], and the in-plane sideways oscillations (present paper). The complete study of the active control of friction by the in-plane oscillations in the sliding direction is still open.

Further generalization of the present work could lead to consideration of contacts under simultaneous oscillations in many directions. An example of such a study carried out in Ref. [14] shows that multi-mode "active control of friction" leads to some qualitatively new effects such as actuation due to symmetry breaking.

Finally, let us mention that the present study can be extended by consideration of the contact dynamics on the time scale that was classified as "microscopic" in the present study. This would lead to non-local (temporal) dependences of the frictional force on the loading, or in other words the kinetics of the coefficient of friction. The basics for such a consideration are already given by Eqs. (9)-(12) but have not been an explicit subject of study in the present paper.

\section{Acknowledgements}

This work was supported in part by the program ACCEL of Japan Science and Technology Agency, the Deutsche Forschungsgemeinschaft and the Ministry of Education of the Russian Federation.

Open Access: The articles published in this journal are distributed under the terms of the Creative Commons Attribution 4.0 International License (http:// creativecommons.org/licenses/by/4.0/), which permits unrestricted use, distribution, and reproduction in any medium, provided you give appropriate credit to the original author(s) and the source, provide a link to the Creative Commons license, and indicate if changes were made.

\section{References}

[1] Brillouin M, Théorie moléculaire du frottement des solides polis, Paris (France): V. Masson, 1899.

[2] Popov V L. Contact Mechanics and Friction. Physical Principles and Applications. 2nd ed. Berlin (Germany): Springer, 2017.

[3] Bowden F P, Tabor D. The Friction and Lubrication of Solids. New York (USA): Oxford University Press, 1950. 
[4] Storck H, Littmann W, Wallaschek J, Mracek M. The effect of friction reduction in presence of ultrasonic vibrations and its relevance to travelling wave ultrasonic motors. Ultrasonics 40(1-8): 379-383 (2002)

[5] Teidelt E, Starcevic J, Popov V L. Influence of ultrasonic oscillation on static and sliding friction. Tribol Lett 48(1): 51-62 (2012)

[6] Milahin N, Li Q, Starčević J. Influence of the normal force on the sliding friction under ultrasonic oscillations. Facta Univ Ser Mech Eng 13(1): 27-32 (2015)

[7] Hoffmann N, Fischer M, Allgaier R, Gaul L. A minimal model for studying properties of the mode-coupling type instability in friction induced oscillations. Mech Res Commun 29(4): 197-205 (2002)

[8] von Wagner U, Hochlenert D, Hagedorn P. Minimal models for disk brake squeal. J Sound Vib 302(3): 527-539 (2007)

[9] Adams G G. Self-excited oscillations of two elastic halfspaces sliding with a constant coefficient of friction. $J$ Appl Mech 62(4): 867-872 (1995)

[10] Comninou M, Dundurs J. Can two solids slide without slipping? Int J Solids Struct 14(4): 251-260 (1978)

[11] Adams G G. Steady sliding of two elastic half-spaces with friction reduction due to interface stick-slip. J Appl Mech 65(2): 470-475 (1998)

[12] Wetter R, Popov V L. The influence of system dynamics on the frictional resistance: insights from a discrete model. Tribol Lett 61(2): 15 (2016)

[13] Popov V L. Oscillation-based methods for actuation and manipulation of nano-objects. AIP Conference Proceedings 1882, 020056 (2017); https://doi.org/10.1063/1.5001635

[14] Popov M, Li Q. Multi-mode active control of friction, dynamic ratchets and actuators. Phys Mesomechan 21(1): (2018)

[15] Teidelt E. Oscillating contacts: friction induced motion and control of friction. Ph.D. Thesis. Berlin (Germany): Technische Universität, 2015.

[16] Wallaschek J. Contact mechanics of piezoelectric ultrasonic motors. Smart Mater Struct 7(3): 369-381 (1998)

[17] Edeler C, Meyer I, Fatikow S. Modeling of stick-slip micro-drives. J Micro-Nano Mechatron 6(3-4): 65-87 (2011)

[18] Mindlin R D, Mason W P, Osmer J F, Deresiewicz H. Effects of an oscillating tangential force on the contact surfaces of elastic spheres. In Proceedings of the 1st US National Congress of Applied Mech., New York, 1952: 203-208.

[19] Gaul L, Lenz J. Nonlinear dynamics of structures assembled by bolted joints. Acta Mech 125(1-4): 169-181 (1997)

[20] Popov M, Popov V L, Pohrt R. Relaxation damping in oscillating contacts. Sci Rep 5: 16189 (2015)
[21] Fridman H D, Levesque P. Reduction of static friction by sonic vibrations. J Appl Phys 30(10): 1572-1575 (1959)

[22] Pohlman R, Lehfeldt E. Influence of ultrasonic vibration on metallic friction. Ultrasonics 4(4): 178-185 (1966)

[23] Godfrey D. Vibration reduces metal to metal contact and causes an apparent reduction in friction. ASLE Trans 10(2): 183-192 (1967)

[24] Lenkiewicz W. The sliding friction process-effect of external vibrations. Wear 13(2): 99-108 (1969)

[25] Tolstoi D M. Significance of the normal degree of freedom and natural normal vibrations in contact friction Wear 10(3): 199-213 (1967)

[26] Starcevic J, Filippov A E. Simulation of the influence of ultrasonic in-plane oscillations on dry friction accounting for stick and creep. Phys Mesomech 15(5-6): 330-332 (2012)

[27] Teidelt E, Willert E, Filippov A E, Popov V L. Modeling of the dynamic contact in stick-slip microdrives using the method of reduction of dimensionality. Phys Mesomech 15(5-6): 287-292 (2012)

[28] Popov M, Popov V L, Popov N V. Reduction of friction by normal oscillations. I. Influence of contact stiffness. Friction 5(1): 45-55 (2017)

[29] Mao X Y, Popov V L, Starcevic J, Popov M. Reduction of friction by normal oscillations. II. In-plane system dynamics. Friction 5(2): 194-206 (2017)

[30] Kado N, Tadokoro C, Nakano K. Measurement error of kinetic friction coefficient generated by frictional vibration. Trans Jpn Soc Mech Eng Ser C 79(803): 2635-2643 (2013)

[31] Kado N, Tadokoro C, Nakano K. Kinetic friction coefficient measured in tribotesting: influence of frictional vibration. Tribol Online 9(2): 63-70 (2014)

[32] Kado N, Sato N, Tadokoro C, Skarolek A, Nakano K. Effect of yaw angle misalignment on brake noise and brake time in a pad-on-disc-type apparatus with unidirectional compliance for pad support. Tribol Int 78: 41-46 (2014)

[33] Kado N, Nakano K. Stabilizing effect of in-plane angular misalignment in 2DOF sliding system with in-plane anisotropic stiffness. Mech Res Commun 84: 14-19 (2017)

[34] Johnson KL. Contact Mechanics. Cambridge (UK): Cambridge University Press, 1985.

[35] Coulomb C A. Theorie des Machines Simple (Theory of Simple Machines). Paris (France): Bachelier, 1821.

[36] Popova E, Popov V L. The research works of Coulomb and Amontons and generalized laws of friction. Friction 3(2): 183-190 (2015)

[37] Popov M. Critical velocity of controllability of sliding friction by normal oscillations in viscoelastic contacts. Facta Univ Ser Mech Eng 14(3): 335-341 (2016) 


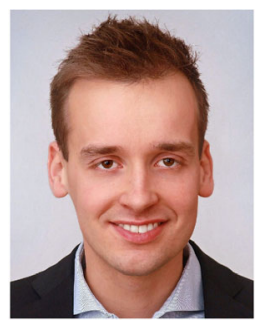

Justus BENAD. He is a research assistant at the Berlin University of Technology where he obtained a Bachelor degree in Aerospace Engineering in 2015 and a Master degree in Engineering Science in 2017. He has work experience in the aerospace industry and in academic research and teaching. He is interested in a wide range of engineering disciplines, among them are numerical simulation methods, software development, tribology,

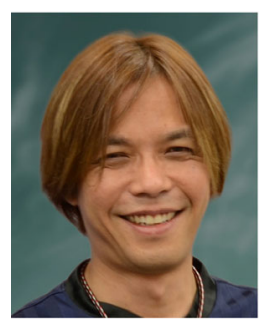

Ken NAKANO. He is a full professor at Faculty of Environment and Information Sciences of Yokohama National University. He studied aeronautics and astronautics at University of Tokyo (1988-1997), and obtained his doctorate from University of Tokyo (1997). He worked at Department of Mechanical Engineering of Saitama University as an assistant professor (1997-2000). After working at Department of Mechanical Engineering of Yokohama National University as an associate professor (20002015), he obtained a full professorship at Yokohama

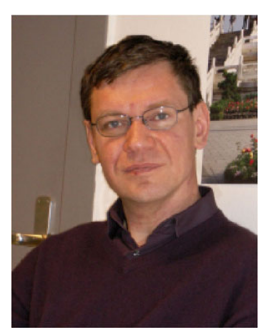

Valentin L. POPOV. He is a full professor at the Berlin University of Technology, studied physics (19761982) and obtained his doctorate in 1985 from the Moscow State Lomonosov University. He worked at the Institute of Strength Physics of the Russian Academy of Sciences. After a guest professorship in the field of theoretical physics at the University of Paderborn (Germany) from 1999 to 2002, he has headed the department of System Dynamics and the Physics of Friction of the Institute of Mechanics at the Berlin University of Technology. His areas of interest include tribology, nanotribology, tribology at low temperatures, biotribology, the influence of friction through ultrasound, numerical simulation of frictional processes, research regarding earthquakes, as well as topics related to materials science such as the fluid and solid mechanics, materials, gas turbines, aircraft design, and areodynamics. Recent projects he has worked on include the simulation of stresses in turbine blades, the preliminary design of a flyingwing aircraft, the development of a hydrofoil control system for racing sailboats, and the simulation of rolling noise of train wheels. He is a scholar of the German National Academic Foundation and has received awards from the Royal Aeronautical Society and the German Aerospace Center.

National University (2015). His areas of interest include tribo-physics (e.g., multiscale and multiphysics of solid friction), tribo-dynamics (e.g., stabilization mechanisms of tribosystems by breaking structural symmetry), and tribo-informatics (e.g., objective evaluation of tactile sensations). In 2015, from Japan Society of Mechanical Engineers (JSME), he was awarded with the JSME Medal for Outstanding Paper on the measurement error of friction coefficient generated by friction-induced vibration. He is the chair of the technical committee on tribo-dynamics of Japanese Society of Tribologists (JAST). He is also a board member of JAST.

mechanics of elastoplastic media with microstructures, strength of metals and alloys, and shape memory alloys. He has published over 100 papers in leading international journals during the past 5 years. He is the author of the book "Contact Mechanics and Friction: Physical principles and applications" which appeared in three German, two English, Chinese, and Russian editions and co-author of the book on "Method of Dimensionality Reduction in Contact Mechanics and Friction" which appeared in German, English and Chinese editions. He is the joint editor of international journals and regularly organizes international conferences and workshops over diverse tribological themes. He is a member of the Scientific Council of the German Tribological Society. He has intensively collaborated with many industrial corporations and possesses experience in implementing the results of scientific research in industrial applications. 


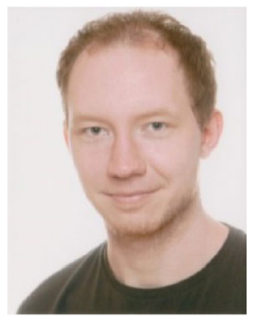

Mikhail POPOV. He received a Bachelor degree in Computer Science from the Freie Universität Berlin in 2011 and a Master degree in Engineering Science from the Tomsk Polytechnic University and the Berlin University of Technology in 2016.
Currently he is a doctoral student at the Berlin University of Technology and a research assistant at the Tomsk Polytechnic University. His research interests include efficient numerical methods in tribology, rubber friction, as well as friction and damping under the influence of oscillations. 